\title{
A comparison of estimated drug costs of potentially inappropriate medications between older patients receiving nurse home visit services and patients receiving pharmacist home visit services: a cross-sectional and propensity score analysis
}

Jun Hamano ${ }^{1 *}$, Sachiko Ozone $e^{2 \dagger}$ and Yasuharu Tokuda ${ }^{3+}$

\begin{abstract}
Background: There have been no multicenter studies that estimated the relations of either nurse or pharmacist home visit program to drug costs of potentially inappropriate medications (PIMs). This study aimed to establish whether patients who used nurse or pharmacist home visit programs (nurse or pharmacist program) had lower drug costs of PIMs than those who did not use nurse or pharmacist programs for older patients living at home.

Methods: This cross-sectional study was conducted in home care settings in Japan, involving 430 patients aged 65 or older, of whom 276 were female. All received regular home visits from five clinics between May and December 2013. After the PIMs were identified with the Screening Tool of Older Persons' potentially inappropriate Prescriptions (STOPP) criteria, we estimated the drug costs based on actual pharmaceutical prices and measured against who using nurse or pharmacist programs after a propensity score weighted adjustment.

Results: Patients who used nurse programs had lower drug cost of PIMs than those who did not use, but it was not significantly different ( $5.9 \pm 13.1$ vs $7.1 \pm 13.9$ USD per month, $P=0.199)$. The cost of PIMs for patients who used pharmacist programs also had no difference. (7.2 \pm 14.5 vs $5.5 \pm 11.5$ USD per month, $P=0.06$ ). In the patient groups who used nurse programs, patients who also used pharmacist programs had significantly higher costs of PIMs than those who used only nurse programs ( $5.5 \pm 13.9$ vs $2.5 \pm 6.0$ USD per month, $P=0.006$ ). In patients group who did not use pharmacist programs, patients who only used nurse programs had significantly lower costs of PIMs than those who did not use nurse programs ( $3.6 \pm 7.7$ vs $5.8 \pm 12.7$ USD per month, $P=0.022$ ).
\end{abstract}

Conclusions: Patients who used nurse program have a trend towards lower drug costs of PIMs than those who used nurse and pharmacist program or pharmacist program alone. Although this study tried to adjust the potential confounders as possible as we could by using propensity score analysis, further studies are needed to confirm our results.

\footnotetext{
*Correspondence: junhamano@md.tsukuba.ac.jp

${ }^{\dagger}$ Equal contributors

'Division of Clinical Medicine, Faculty of Medicine, University of Tsukuba,

Tsukuba, Japan

Full list of author information is available at the end of the article
} 


\section{Background}

Prescription medications are an essential component of the care of older patients. Although some medications can cure and prevent disease, inappropriate medication may have detrimental effects. Potentially inappropriate medications (PIMs) have been defined as medications that carry more risks than benefits, those with clinically significant drug-drug or drug-disease interactions [1,2].

Several studies have shown that PIMs among older patients are correlated with increased adverse drug reactions (ADRs) [3-6], health care utilization [7-9], death [10,11], poor adherence [12,13] and greater economic burden $[7,14,15]$. The cost of potentially inappropriate medications is estimated to be high [16-18], and this has become an important public health issue worldwide [2]. PIMs among older home care patients are common [19], and an important issue in developed countries with an aging population, such as Japan, where the number of older home care patients is predicted to increase rapidly in the future.

Several tools are used to detect PIMs in older people [20], such as the Beers' criteria [21], the Screening Tool of Older Persons' potentially inappropriate Prescriptions (STOPP) [22,23] and the Improved Prescribing in the Elderly Tool (IPET) [24]. Recent studies showed that the STOPP criteria have high sensitivity for detecting potential drug-related problems [25] and are more sensitive in detecting PIMs than the Beers' criteria [26].

Several intervention studies have revealed that a multidisciplinary approach for older patients could reduce the number of patients with PIMs $[27,28]$ and detect a high proportion of clinically relevant DRPs $[29,30]$. One previous study conducted in home care settings revealed that a medication review by a community pharmacist could reduce the number of medications, but it was not clear about the relations of pharmacist intervention to drug costs of PIMs [31]. As far as we know, there has been no multicenter study that estimated the relations of either nurse or pharmacist home visit program to drug cost of PIMs identified by the STOPP criteria for older patients in home care settings. This study aimed to validate whether the patients who using either nurse or pharmacist home visit programs had lower drug costs of potentially inappropriate medication based on actual pharmaceutical prices than who not using nurse or pharmacist home visit programs for older patients in a home care setting.

\section{Methods}

This cross-sectional study was conducted at five clinics in Japan between May and December 2013. These clinics provide primary care by ambulatory service and home visit services for community residents, and each clinics collaborate with home visit nurses and pharmacists depending on the situation. None of these doctors were familiar with the STOPP criteria at the starting time of study. In general, nurse home visit programs in Japan provide hands-on care at home, for example to help bathing, to promote physical activity and to coordinate living environment. Pharmacist home visit programs in Japan usually consult with patient regarding expected or unexpected effects of drugs and monitor the adherence of prescription at home. The frequency of their home visit depended on patients' conditions and needs for care, typically twice or four times a month by nurse and a once or twice a month by pharmacist.

Although, the primary care doctors responsible for care of individual patients typically recommend using the home visit program and patients or their family could decide to use or not, the patients and their family could use the programs whenever they requested.

The study was approved by the ethics committee of the Mito Kyodo General Hospital and was conducted according to the principles expressed in the Declaration of Helsinki. We included all patients who were 65 years or older and who satisfied our inclusion criteria, which were:

1) patients received home visit services regularly by their doctors at least twice a month for over a month, and

2) patients had been regularly prescribed medications by the clinic, excluding topical drugs.

We used medical records to collect patients' background information, which included age, sex, estimated glomerular filtration rate (eGFR: $\mathrm{ml} / \mathrm{min}$ ), serum albu$\min (\mathrm{mg} / \mathrm{dl})$, availability of overview of ambulation and drug use, underlying medical conditions, whether they lived with or without family, and whether they used a home visit nurse or pharmacist.

We had collected the copies of prescription contents by single monthly basis during the survey period which was sent from each clinic and confirmed the medication.

We defined PIMs as having occurred when at least one of the original STOPP criteria was met (Table 1). We calculated the total monthly drug cost of all patients and the monthly drug cost of PIMs. We estimated the drug cost based on actual pharmaceutical prices listed by the regulatory committee at the Ministry of Health, Labour and Welfare, Japanese government.

\section{Statistical methods}

We used Student's $t$ test for continuous variables and Pearson's $\chi^{2}$ test or Fisher's exact test for categorical variables to test for significant associations between patients' baseline characteristics and the use of the home visit nurse or pharmacist. We used a propensity score weighting technique to assess the association between the monthly drug cost of PIMs and the use of the home visit nurse or pharmacist and to adjust for confounding factors. 
Table 1 STOPP screening criteria [22]

\section{STOPP criteria}

A. Cardiovascular system

1

2

3

7

9

10

B. CNS and psychotropic drugs

C. Gastrointestinal system

1

4

D. Respiratory system

1

E. Musculoskeletal system

1
Digoxin at a long-term dose $>125 \mathrm{mg} / \mathrm{d}$ with impaired renal function Loop diuretic as first-line monotherapy for hypertension or for dependent ankle edema only Thiazide diuretic with a history of gout Noncardioselective b-blocker with chronic obstructive pulmonary disease b-blocker in combination with verapamil Use of diltiazem or verapamil with New York Heart Association class 3 or 4 heart failure Calcium-channel blockers with chronic constipation Use of aspirin and warfarin in combination without histamine $\mathrm{H} 2$ receptor antagonist (except cimetidine) or proton pump inhibitor

Dipyridamole as monotherapy for cardiovascular secondary prevention Aspirin with no history of coronary, cerebral or peripheral vascular symptoms or occlusive arterial event or with a past history of peptic ulcer disease without histamine $\mathrm{H} 2$ receptor antagonist or proton pumpinhibitor or at dose $>150 \mathrm{mg} / \mathrm{d}$ or to treat dizziness not clearly attributed to cerebrovascular disease Warfarin for first, uncomplicated deep venous thrombosis for longer than 6-month duration or for first, uncomplicated pulmonary embolus for longer than 12-month duration Aspirin, clopidogrel, dipyridamole, or warfarin with concurrent bleeding disorder

Tricyclic antidepressants with dementia or with glaucoma or with cardiac conductive abnormalities or with constipation or with prostatism or prior history of urinary retention or with an opiate or calcium-channel blocker Long-term (>3 month) use of long-acting benzodiazepines, and with long-acting metabolites Long-term (>1 month) neuroleptics as long-term hypnotics or in those with parkinsonism Phenothiazines in patients with epilepsy Anticholinergics to treat extrapyramidal side effects of neuroleptic medications Selective serotonin reuptake inhibitors with a history of clinically significant hyponatremia (noniatrogenic hyponatremia $<130 \mathrm{mmol} / \mathrm{L}$ within the previous 2 months)

Prolonged use (>1 week) of first-generation antihistamines

Diphenoxylate, loperamide or codeine phosphate for treatment of diarrhea of unknown cause or severe infective gastroenteritis

Prochlorperazine or metoclopramide with parkinsonism

Proton pump inhibitors for peptic ulcer disease at full therapeutic dosage for $>8$ weeks Anticholinergic antispasmodic drugs with chronic constipation

Theophylline as monotherapy for chronic obstructive pulmonary disease Systemic corticosteroids instead of inhaled corticosteroids for maintenance therapy in moderate-to-severe chronic obstructive pulmonary disease Nebulized ipratropium with glaucoma

NSAID with history of peptic ulcer disease or gastrointestinal bleeding, unless with concurrent histamine $\mathrm{H} 2$ receptor antagonist, proton pump inhibitor, or misoprostol NSAID with moderate-to-severe hypertension or with heart failure 
Table 1 STOPP screening criteria [22] (Continued)

\begin{tabular}{|c|c|}
\hline 3 & Long-term use of NSAID (>3 months) for relief of mild joint pain in osteoarthritis \\
\hline 4 & Warfarin and NSAID together \\
\hline 5 & NSAID with chronic renal failure \\
\hline \multirow[t]{2}{*}{6} & Long-term corticosteroids (>3 months) as monotherapy for rheumatoid arthritis \\
\hline & or osteoarthritis \\
\hline \multirow[t]{2}{*}{7} & Long-term NSAID or colchicine for chronic treatment of gout where there is \\
\hline & no contraindication to allopurinol \\
\hline \multicolumn{2}{|c|}{ F. Urogenital system } \\
\hline \multirow[t]{2}{*}{1} & Bladder antimuscarinic drugs with dementia or with chronic glaucoma \\
\hline & or with chronic constipation or with chronic prostatism \\
\hline 2 & a-blockers in males with frequent incontinence \\
\hline \multicolumn{2}{|c|}{ G. Endocrine system } \\
\hline 1 & Glibenclamide or chlorpropamide with type 2 diabetes mellitus \\
\hline 2 & b-blockers in those with diabetes mellitus and frequent hypoglycemic episodes \\
\hline 3 & Estrogens with a history of breast cancer or venous thromboembolism \\
\hline 4 & Estrogens without progestogen in patients with intact uterus \\
\hline \multicolumn{2}{|c|}{$\begin{array}{l}\text { H. Drugs that adversely affect those prone to falls } \\
\text { (at least } 1 \text { fall in past } 3 \text { months) }\end{array}$} \\
\hline 1 & Benzodiazepines \\
\hline 2 & Neuroleptic drugs \\
\hline 3 & First-generation antihistamines \\
\hline 4 & Vasodilator drugs known to cause hypotension in those with persistent postural hypotension \\
\hline \multicolumn{2}{|c|}{ I. Analgesic drugs } \\
\hline 1 & Use of long-term powerful opiates as first-line therapy for mild-to-moderate pain \\
\hline \multirow[t]{2}{*}{2} & Regular opiates for more than 2 weeks in those with chronic constipation \\
\hline & without concurrent use of laxatives \\
\hline \multirow[t]{2}{*}{3} & Long-term opiates in those with dementia unless indicated for palliative care \\
\hline & or management of moderate-to-severe chronic pain syndrome \\
\hline \multicolumn{2}{|c|}{ J. Duplicate drug classes } \\
\hline \multirow[t]{2}{*}{1} & Any regular duplicate drug class prescription, such as two concurrent opiates, NSAIDs, \\
\hline & serotonin-specific reuptake inhibitors, loop diuretics, and ACE inhibitors \\
\hline
\end{tabular}

For the propensity score analysis, we selected the variables as those that 1 ) were hypothesized to be strongly associated with the use of the home visit nurse or pharmacist and PIMs, and 2) were hypothesized to be associated with PIMs, and excluded those that 1 ) were associated with other aspects as well as the use of the home visit nurse and pharmacist, 2) were affected by the use of the home visit nurse or pharmacist, 3) perfectly predict the use of the home visit nurse and pharmacist [32].

We then used logistic regression to calculate the propensity score, categorizing the patient age into three groups: $65-74,75-84$, and $>85$ years.
The logistic regression model for the propensity score for the use of the nurse home visit programs (PS for nurse) included age category, sex, availability of overview on ambulation and drug use, whether they lived with or without family, the use of the home visit pharmacist, number of prescriptions and underlying medical conditions (constipation, hypertension, dementia, cerebral infarction/ transient ischemic attack, diabetes mellitus, atrial fibrillation, progressive malignancy, hyperuricemia/gout, heart failure, dyslipidemia, Parkinson's Disease/Parkinson's syndrome, osteoporosis, chronic obstructive pulmonary disease, peripheral artery occlusive disease, cerebral/subarachnoid hemorrhage, osteoarthritis) as variables. 
Table 2 Patient background $(n=430)$

\begin{tabular}{|c|c|c|c|c|c|c|c|c|c|c|c|c|c|}
\hline & \multirow{3}{*}{$\begin{array}{l}\text { All patients } \\
(n=430) ; \\
n(\%)\end{array}$} & \multicolumn{6}{|c|}{ Before propensity score weighted adjustment } & \multicolumn{6}{|c|}{ After propensity score weighted adjustment } \\
\hline & & \multicolumn{3}{|c|}{ Using nurse home visit programs } & \multicolumn{3}{|c|}{$\begin{array}{l}\text { Using pharmacist } \\
\text { home visit programs }\end{array}$} & \multicolumn{3}{|c|}{$\begin{array}{l}\text { Using nurse home visit } \\
\text { programs (weighted by } \\
\text { PS for nurse) }\end{array}$} & \multicolumn{3}{|c|}{$\begin{array}{l}\text { Using pharmacist home } \\
\text { visit programs (weighted } \\
\text { by PS for pharmacist) }\end{array}$} \\
\hline & & $\begin{array}{l}\text { Patients who } \\
\text { used nurse } \\
\text { home visit } \\
\text { programs } \\
(n=203) ; \\
n(\%)\end{array}$ & $\begin{array}{l}\text { Patients who } \\
\text { did not used } \\
\text { nurse home } \\
\text { visit programs } \\
\text { ( } \mathrm{n}=227) ; \\
\text { n (\%) }\end{array}$ & $\begin{array}{l}\mathrm{P}- \\
\text { value }\end{array}$ & $\begin{array}{l}\text { Patients } \\
\text { who used } \\
\text { pharmacist } \\
\text { home visit } \\
\text { programs } \\
(\mathrm{n}=182) ; \\
\mathrm{n}(\%)\end{array}$ & $\begin{array}{l}\text { Patients who } \\
\text { did not used } \\
\text { pharmacist } \\
\text { home visit } \\
\text { programs } \\
\text { ( } \mathrm{n}=248) ; \\
\mathrm{n}(\%)\end{array}$ & $\begin{array}{l}\mathrm{P} \text { - } \\
\text { value }\end{array}$ & $\begin{array}{l}\text { Patients who } \\
\text { used nurse } \\
\text { home visit } \\
\text { programs (\%) }\end{array}$ & $\begin{array}{l}\text { Patients } \\
\text { who did } \\
\text { not used } \\
\text { nurse home } \\
\text { visit programs } \\
\text { (\%) }\end{array}$ & $\begin{array}{l}\mathrm{P} \text { - } \\
\text { value }\end{array}$ & $\begin{array}{l}\text { Patients } \\
\text { who used } \\
\text { pharmacist } \\
\text { home visit } \\
\text { programs (\%) }\end{array}$ & $\begin{array}{l}\text { Patients who } \\
\text { did not used } \\
\text { pharmacist } \\
\text { home visit } \\
\text { programs (\%) }\end{array}$ & $\begin{array}{l}P \text { - } \\
\text { value }\end{array}$ \\
\hline Gender & & & & 0.013 & & & 0.073 & & & 0.924 & & & 0.950 \\
\hline Male & $154(35.8 \%)$ & $85(41.9 \%)$ & $69(30.4 \%)$ & & $74(40.7 \%)$ & $80(32.3 \%)$ & & $(34.3 \%)$ & $(34.0 \%)$ & & $(35.6 \%)$ & $(35.4 \%)$ & \\
\hline Female & $276(64.2 \%)$ & $118(58.1 \%)$ & $158(69.6 \%)$ & & $\begin{array}{l}108 \\
(59.3 \%)\end{array}$ & $168(67.7 \%)$ & & $(65.7 \%)$ & $(66.0 \%)$ & & $(64.4 \%)$ & $(64.6 \%)$ & \\
\hline $\begin{array}{l}\text { Mean age (years } \pm \\
\text { standard deviation) }\end{array}$ & $85.0 \pm 8.3$ & $84.3 \pm 8.6$ & $85.7 \pm 8.0$ & 0.07 & $84.7 \pm 8.1$ & $85.2 \pm 8.4$ & 0.523 & $85.5 \pm 8.5$ & $84.9 \pm 8.1$ & 0.298 & $85.3 \pm 8.3$ & $85.0 \pm 8.2$ & 0.575 \\
\hline Age (years) & & & & 0.004 & & & 0.177 & & & 0.909 & & & 0.976 \\
\hline $65-74$ & $55(12.8 \%)$ & $29(14.3 \%)$ & $26(11.5 \%)$ & & $22(12.1 \%)$ & $33(13.3 \%)$ & & $(12.0 \%)$ & $(12.1 \%)$ & & $(12.9 \%)$ & $(12.4 \%)$ & \\
\hline 75-84 & $126(29.3 \%)$ & $73(36.0 \%)$ & $53(23.3 \%)$ & & 62 (34.1\%) & 64 (25.8\%) & & (30.6\%) & (31.9\%) & & $(28.8 \%)$ & $(29.0 \%)$ & \\
\hline$\geq 85$ & 249 (57.9\%) & $101(49.8 \%)$ & $148(65.2 \%)$ & & 98 (53.8\%) & $151(60.9 \%)$ & & (56.0\%) & (56.0\%) & & $(58.3 \%)$ & $(58.5 \%)$ & \\
\hline $\begin{array}{l}\text { Independent } \\
\text { ambulation }\end{array}$ & $199(46.3 \%)$ & 71 (35.0\%) & $128(56.4 \%)$ & $<0.001$ & 91 (50.0\%) & $108(43.5 \%)$ & 0.185 & $(45.3 \%)$ & (45.6\%) & 0.925 & $(45.9 \%)$ & $(46.1 \%)$ & 0.934 \\
\hline $\begin{array}{l}\text { Independent } \\
\text { drug overview }\end{array}$ & 115 (26.7\%) & $44(21.7 \%)$ & 71 (31.3\%) & 0.025 & $54(29.7 \%)$ & 61 (24.6\%) & 0.24 & $(22.8 \%)$ & (24.8\%) & 0.482 & $(27.4 \%)$ & $(26.7 \%)$ & 0.812 \\
\hline Living with family & 381 (88.6\%) & $190(93.6 \%)$ & $191(84.1 \%)$ & 0.002 & $\begin{array}{l}155 \\
(85.2 \%)\end{array}$ & $226(91.1 \%)$ & 0.054 & (87.3\%) & (88.4\%) & 0.626 & $(88.7 \%)$ & (88.1\%) & 0.774 \\
\hline $\begin{array}{l}\text { Using home visit } \\
\text { programs by nurse }\end{array}$ & $203(47.2 \%)$ & - & - & - & 78 (42.9\%) & $125(50.4 \%)$ & 0.121 & - & - & - & $(47.7 \%)$ & $(47.1 \%)$ & 0.855 \\
\hline $\begin{array}{l}\text { Using home visit } \\
\text { programs by } \\
\text { pharmacist }\end{array}$ & $182(42.3 \%)$ & 78 (38.4\%) & $104(45.8 \%)$ & 0.121 & - & - & - & $(41.5 \%)$ & $(42.2 \%)$ & 0.844 & - & - & - \\
\hline \multicolumn{14}{|l|}{$\begin{array}{l}\text { Underlying medical } \\
\text { conditions }\end{array}$} \\
\hline Constipation & 243 (56.5\%) & $113(55.7 \%)$ & $130(57.3 \%)$ & 0.738 & $\begin{array}{l}115 \\
(63.2 \%)\end{array}$ & $128(51.6 \%)$ & 0.017 & $59.6 \%$ & $59.4 \%$ & 0.955 & $55.4 \%$ & $56.2 \%$ & 0.818 \\
\hline Hypertension & $228(53.0 \%)$ & $84(41.4 \%)$ & $144(63.4 \%)$ & $<0.001$ & $\begin{array}{l}101 \\
(55.5 \%)\end{array}$ & $127(51.2 \%)$ & 0.379 & $49.5 \%$ & $51.8 \%$ & 0.513 & $52.8 \%$ & $53.2 \%$ & 0.907 \\
\hline \multirow[t]{2}{*}{ Dementia } & $218(50.7 \%)$ & 87 (42.9\%) & $131(57.7 \%)$ & 0.002 & 89 (48.9\%) & $129(52.0 \%)$ & 0.523 & $52.5 \%$ & $54.2 \%$ & 0.601 & $50.9 \%$ & $51.5 \%$ & 0.860 \\
\hline & 104 (24.2\%) & 45 (22.2\%) & $59(26.0 \%)$ & 0.355 & $44(24.2 \%)$ & $60(24.2 \%)$ & 0.997 & $26.8 \%$ & $24.5 \%$ & 0.456 & $24.7 \%$ & $25.1 \%$ & 0.906 \\
\hline
\end{tabular}


Table 2 Patient background $(n=430)$ (Continued)

\begin{tabular}{|c|c|c|c|c|c|c|c|c|c|c|c|c|c|}
\hline \multicolumn{14}{|l|}{$\begin{array}{l}\text { Cerebral infarction/ } \\
\text { transient ischemic } \\
\text { attack }\end{array}$} \\
\hline Osteoporosis & 95 (22.1\%) & 38 (18.7\%) & $57(25.1 \%)$ & 0.111 & 47 (25.8\%) & 48 (19.4\%) & 0.11 & $26.0 \%$ & $22.9 \%$ & 0.299 & $22.1 \%$ & $22.5 \%$ & 0.898 \\
\hline Diabetes mellitus & $82(19.1 \%)$ & $33(16.3 \%)$ & 49 (21.6\%) & 0.16 & $43(23.6 \%)$ & 39 (15.7\%) & 0.039 & $20.2 \%$ & $19.1 \%$ & 0.691 & $19.2 \%$ & $19.7 \%$ & 0.852 \\
\hline $\begin{array}{l}\text { Coronary artery } \\
\text { disease }\end{array}$ & $59(13.7 \%)$ & $27(13.3 \%)$ & $32(14.1 \%)$ & 0.811 & $28(15.4 \%)$ & $31(12.5 \%)$ & 0.39 & $13.3 \%$ & $15.3 \%$ & 0.410 & $11.8 \%$ & $13.1 \%$ & 0.544 \\
\hline Atrial fibrillation & $55(12.8) \%$ & $29(14.3 \%)$ & $26(11.5 \%)$ & 0.38 & $27(14.8 \%)$ & $28(11.3 \%)$ & 0.277 & $12.9 \%$ & $12.3 \%$ & 0.776 & $12.7 \%$ & $12.2 \%$ & 0.816 \\
\hline Dyslipidemia & $51(11.9 \%)$ & $17(8.4 \%)$ & $34(15.0 \%)$ & 0.034 & $27(14.8 \%)$ & $24(9.7 \%)$ & 0.102 & $9.9 \%$ & $11.8 \%$ & 0.358 & $12.2 \%$ & $12.6 \%$ & 0.847 \\
\hline $\begin{array}{l}\text { Benign prostatic } \\
\text { hypertrophy }\end{array}$ & 47 (10.9\%) & $24(11.8 \%)$ & $23(10.1 \%)$ & 0.575 & $22(12.1 \%)$ & $25(10.1 \%)$ & 0.51 & $10.1 \%$ & $11.6 \%$ & 0.485 & $9.9 \%$ & $10.8 \%$ & 0.685 \\
\hline $\begin{array}{l}\text { Progressive } \\
\text { malignancy }\end{array}$ & $34(7.9 \%)$ & $23(11.3 \%)$ & $11(4.8 \%)$ & 0.013 & $14(7.7 \%)$ & $20(8.1 \%)$ & 0.888 & $7.5 \%$ & $7.1 \%$ & 0.807 & $7.2 \%$ & $7.7 \%$ & 0.751 \\
\hline Hyperuricemia/gout & $32(7.4 \%)$ & $9(4.4 \%)$ & $23(10.1 \%)$ & 0.025 & 19 (10.4\%) & $13(5.2 \%)$ & 0.042 & $9.2 \%$ & $7.8 \%$ & 0.473 & $8.1 \%$ & $8.2 \%$ & 0.951 \\
\hline Heart failure & $30(7.0 \%)$ & $17(8.4 \%)$ & $13(5.7 \%)$ & 0.282 & 14 (7.7\%) & $16(6.5 \%)$ & 0.618 & $5.0 \%$ & $7.3 \%$ & 0.163 & $6.2 \%$ & $5.9 \%$ & 0.815 \\
\hline $\begin{array}{l}\text { Parkinson's Disease/ } \\
\text { Parkinson's syndrome }\end{array}$ & $28(6.5 \%)$ & 14 (6.9\%) & 14 (6.2\%) & 0.76 & 13 (7.1\%) & $15(6.0 \%)$ & 0.649 & $7.5 \%$ & $7.8 \%$ & 0.866 & $6.2 \%$ & $6.6 \%$ & 0.840 \\
\hline $\begin{array}{l}\text { Chronic obstructive } \\
\text { pulmonary disease }\end{array}$ & 27 (6.3\%) & 19 (9.4\%) & $8(3.5 \%)$ & 0.013 & 17 (9.3\%) & 10 (4.0\%) & 0.025 & $5.9 \%$ & $3.8 \%$ & 0.156 & $6.0 \%$ & $5.2 \%$ & 0.592 \\
\hline $\begin{array}{l}\text { Peripheral artery occlusive } \\
\text { disease }\end{array}$ & 27 (6.3\%) & $7(3.4 \%)$ & $20(8.8 \%)$ & 0.022 & 16 (8.8\%) & 11 (4.4\%) & 0.066 & $4.0 \%$ & $5.7 \%$ & 0.253 & $6.0 \%$ & $5.6 \%$ & 0.816 \\
\hline $\begin{array}{l}\text { Cerebral/ } \\
\text { subarachnoid hemorrhage }\end{array}$ & $26(6.0 \%)$ & $11(5.4 \%)$ & 15 (6.6\%) & 0.606 & 17 (9.3\%) & $9(3.6 \%)$ & 0.014 & $5.2 \%$ & $6.4 \%$ & 0.452 & $6.0 \%$ & $6.3 \%$ & 0.846 \\
\hline Osteoarthritis & $21(4.9 \%)$ & $6(3.0 \%)$ & $15(6.6 \%)$ & 0.079 & 9 (4.9\%) & $12(4.8 \%)$ & 0.96 & $5.6 \%$ & $5.0 \%$ & 0.658 & $5.1 \%$ & $4.9 \%$ & 0.919 \\
\hline $\begin{array}{l}\text { Number of } \\
\text { prescriptions } \\
\text { (mean } \pm \text { standard } \\
\text { deviation) }\end{array}$ & $6.1 \pm 3.0$ & $6.3 \pm 3.0$ & $5.8 \pm 3.0$ & 0.065 & $6.6 \pm 3.1$ & $5.6 \pm 2.8$ & $<0.001$ & $6.2 \pm 3.0$ & $6.0 \pm 3.1$ & 0.427 & $5.9 \pm 3.0$ & $6.0 \pm 3.0$ & 0.879 \\
\hline
\end{tabular}


Table 3 Most frequent prescriptions resulting in PIMs

\begin{tabular}{|c|c|c|}
\hline & Prescription & $(n=430) ; n(\%)$ \\
\hline 1 & Calcium-channel blockers with chronic constipation & $74(17.2 \%)$ \\
\hline 2 & Long-term use of NSAID (>3 months) for relief of mild joint pain in osteoarthritis & $16(3.7 \%)$ \\
\hline 3 & Long-term (>1 month) use of long-acting benzodiazepines, and with long-acting metabolites & $15(3.5 \%)$ \\
\hline 4 & NSAID with moderate-to-severe hypertension or with heart failure & $14(3.3 \%)$ \\
\hline 5 & Loop diuretic as first-line monotherapy for hypertension or for dependent ankle edema only & $13(3.0 \%)$ \\
\hline 6 & $\begin{array}{l}\text { Any regular duplicate drug class prescription, such as two concurrent opiates, NSAIDs, } \\
\text { serotonin-specific reuptake inhibitors, loop diuretics, and ACE inhibitors }\end{array}$ & $11(2.6 \%)$ \\
\hline 7 & Benzodiazepines for patients who have had at least 1 fall in the past 3 months & $10(2.3 \%)$ \\
\hline
\end{tabular}

We also developed the propensity score for the use of the pharmacist home visit programs (PS for pharmacist) using a logistic regression model that included the same variables as for the nurse home visit programs, except that use of the pharmacist home visit programs was changed to use of the nurse home visit program.

To examine the association between the monthly drug cost of PIMs and use of the nurse or pharmacist home visit programs, we used a multivariate logistic regression analysis and included use of the home visit programs with the propensity score as variables. All analyses were conducted using SPSS-J (ver. 22.0; IBM, Tokyo, Japan).

\section{Results}

\section{Demography}

We included 430 patients in this study, of whom 276 were female. Table 2 shows detailed patient background information. The mean patient age was $85.0 \pm 8.3$ years. The main underlying medical conditions were constipation in 243 patients (56.5\%), hypertension in 228 patients $(53.0 \%)$, and dementia in 218 patients (50.7\%). Nearly half, 203 patients (47.2\%), had used the nurse home visit programs and $182(42.3 \%)$ had used the pharmacist home visit programs. Almost one fifth, 78 patients (18.1\%), had used both nurse and pharmacist home visit programs (Table 2). Over one quarter, 123 patients (28.6\%), had not used either program.
Propensity score weighting successfully balanced the observed differences in patient background and the use of the two home visit programs (Table 2).

\section{Specific prescriptions of PIMs}

By the STOPP criteria, $34.0 \%$ of the study population received at least one PIM. We found that the most common prescriptions resulting in PIMs were (a) calcium-channel blockers in patients with chronic constipation (74 patients, 17.2\%), (b) long-term use of NSAIDs for relief of mild joint pain in osteoarthritis (16 patients, 3.7\%), (c) long-term use of long-acting benzodiazepines (15 patients, 3.5\%), and (d) NSAIDs in patients with moderate-to-severe hypertension or with heart failure (14 patients, 3.3\%) (Table 3).

\section{The cost of PIMs after propensity score weighted adjustment}

After propensity score weighted adjustment, we compared the total cost of PIMs per patient per month in USD (100 Japanese Yen = 1 USD) in all patients and each subgroup (Table 4,5). Although it was not significantly different, patients who used the pharmacist home visit programs had higher drug costs of PIMs (7.2 \pm 14.5 vs $5.5 \pm$ 11.5 USD per month, $\mathrm{P}=0.06$ ) (Table 4). On the other hand, the drug cost of PIMs for patients who used the nurse home visit programs was lower than those who did not use, but it was not significantly different $(5.9 \pm 13.1$ vs

Table 4 The cost of PIMs in patients group who used nurse home visit or not

\begin{tabular}{|c|c|c|c|c|c|c|}
\hline \multicolumn{7}{|c|}{ (Total cost of PIMs per patient per month in USD) } \\
\hline & \multirow[b]{2}{*}{$\mathbf{n}$} & \multicolumn{5}{|c|}{ Using pharmacist home visit programs (weighted by PS for pharmacist) } \\
\hline & & $\begin{array}{l}\text { Patients who used } \\
\text { pharmacist home visit } \\
\text { programs }(n=182)\end{array}$ & $\begin{array}{l}\text { Patients who did not } \\
\text { use pharmacist home } \\
\text { visit programs }(n=248)\end{array}$ & $\begin{array}{l}\text { Difference between } \\
\text { two groups }\end{array}$ & $95 \% \mathrm{Cl}$ & P-value \\
\hline All patients $^{\dagger}$ & 430 & $7.2 \pm 14.5$ & $5.5 \pm 11.5$ & -1.7 & $-3.5-0.07$ & 0.06 \\
\hline $\begin{array}{l}\text { Patients who used nurse home } \\
\text { visit programs }\end{array}$ & 203 & $5.5 \pm 13.9$ & $2.5 \pm 6.0$ & -2.9 & $-5.0--0.83$ & 0.006 \\
\hline $\begin{array}{l}\text { Patients who did not use nurse } \\
\text { home visit programs }^{\dagger}\end{array}$ & 227 & $8.7 \pm 15.7$ & $8.1 \pm 14.2$ & -0.66 & $-3.4-2.1$ & 0.64 \\
\hline
\end{tabular}

${ }^{\dagger}$ drug cost (USD) \pm standard deviation. 
Table 5 The cost of PIMs in patients group who used pharmacist home visit or not

(Total cost of PIMs per patient per month in USD)

\begin{tabular}{|c|c|c|c|c|c|c|}
\hline & \multirow[b]{2}{*}{$\mathbf{n}$} & \multicolumn{5}{|c|}{ Using nurse home visit programs (weighted by PS for nurse) } \\
\hline & & $\begin{array}{l}\text { Patients who used } \\
\text { nurse home visit } \\
\text { programs }(n=203)\end{array}$ & $\begin{array}{l}\text { Patients who did not } \\
\text { use nurse home visit } \\
\text { programs ( } n=227)\end{array}$ & $\begin{array}{l}\text { Difference } \\
\text { between } \\
\text { two groups }\end{array}$ & $95 \% \mathrm{Cl}$ & P-value \\
\hline All patients $^{\dagger}$ & 430 & $5.9 \pm 13.1$ & $7.1 \pm 13.9$ & 1.2 & $-0.63-3.0$ & 0.199 \\
\hline Patients who used pharmacist home visit programs ${ }^{\dagger}$ & 182 & $9.1 \pm 17.8$ & $8.8 \pm 15.3$ & -0.22 & $-3.7-3.2$ & 0.898 \\
\hline Patients who did not use pharmacist home visit programs ${ }^{\dagger}$ & 248 & $3.6 \pm 7.7$ & $5.8 \pm 12.7$ & 2.2 & $0.31-4.0$ & 0.022 \\
\hline
\end{tabular}

${ }^{\dagger}$ drug cost (USD) \pm standard deviation.

$7.1 \pm$ 13.9 USD per month, $\mathrm{P}=0.199$ ) (Table 5). In the patient groups who used nurse home visiting programs, patients who also used pharmacist home visiting programs had significantly higher costs of PIMs than those who used only nurse home visit programs $(5.5 \pm 13.9$ vs 2.5 \pm 6.0 USD per month, $\mathrm{P}=0.006$ ) (Table 4). In the patient groups who did not use pharmacist home visit programs, patients who only used nurse home visit programs had significantly lower costs of PIMs than those who did not use nurse home visit programs (3.6 \pm 7.7 vs $5.8 \pm 12.7$ USD per month, $\mathrm{P}=0.022$ ) (Table 5).

\section{Discussion}

As far as we know, this is the first study to estimate the relations of nurse and pharmacist home visit program to drug costs of PIMs identified by the STOPP criteria for older patients in home care settings.

The important finding of this study is that those who used nurse home visit program have a trend towards lower drug costs of PIMs than those who used nurse and pharmacist home visit program or pharmacist home visit program alone.

There are several explanations about this finding. First, pharmacist home visit programs tend to involve patients with complicated conditions and PIMs might have been actually appropriate in these circumstances.

Second, although pharmacists understood the concept and meaning of PIMs, pharmacist home visit programs may be more difficult to help for improving the prescription than nurse home visit programs. This hypothesis is based on the situation of pharmacist home visit programs in Japan. In general, pharmacist home visit programs in Japan are expected to encourage to keep the drug adherence and to identify the symptoms as possible drug side effects. On the other hand, pharmacists of home visit programs usually did not receive the details of patients' medical history from doctors and were not expected to give advice to doctors about prescription, although nurse home visit program usually is provided about the details of patients' medical history and often be required to give advice to their patients about prescriptions from doctors in Japan.

Third, there might have been other unidentified confounding variables that might affect the effectiveness of home visit programs to the drug costs of PIMs. We need to conduct further research to reveal factors, which may affect the drug costs of PIMs in home care setting prospectively.

This study had four main limitations. First, we might not be able to assess the all of potential confounders that might affect the drug cost of PIMs in home care setting and the effectiveness of home visit program.

Second, because of its cross-sectional nature, there might have been several potential confounders, we could assess in this study, which could affect the drug costs of PIMs and the effectiveness of home visit program. We performed our analysis to adjust the potential confounders as possible as we could by using propensity scores.

Third, our study sample may not be representative of older home care patients, because it was carried out at only a few institutions in Japan. Further work is needed to carry out a larger study with the greater number of institutions in Japan and other countries.

Finally, we cannot draw conclusions about the effectiveness of nurse and pharmacist home visit program to drug cost of PIMs, because our study was not an intervention study. We would need to carry out further research, including a longitudinal intervention study, to assess the effectiveness of nurse and pharmacist home visit program to drug cost of PIMs.

\section{Conclusion}

In conclusion, those who used nurse home visit program have a trend towards lower drug costs of PIMs than those who used nurse and pharmacist home visit program or pharmacist home visit program alone. Although, this study aimed to adjust the potential confounders as possible as we could by using propensity score analysis, caution may be needed about interpretation of this study. Further research is needed to consider all of potential confounders associated with the drug costs of PIMs in home care setting and using home visit program. 


\section{Competing interests}

The authors declare that they have no competing interests.

\section{Authors' contributions}

$\mathrm{JH}$ and $\mathrm{YT}$ participated in the design of the study and helped to perform the statistical analysis and draft the manuscript. SO contributed to interpretation of the data and manuscript revisions. All authors read and approved the final manuscript.

\section{Acknowledgments}

Research funds for this study were provided by a Clinical Research Grant from St. Luke's Life Science Institute. We would also like to express our gratitude to Kazuhiro Hisajima, MD, Shohei Kawagoe, MD, Hiroyuki Beniya, MD, and Yasuyuki Arai, MD for data collection.

\section{Author details}

'Division of Clinical Medicine, Faculty of Medicine, University of Tsukuba, Tsukuba, Japan. ${ }^{2}$ Primary Care and Medical Education, Graduate School of Comprehensive Human Sciences, University of Tsukuba, Tsukuba, Japan. 3Japan Community Healthcare Organization, 3-22-12 Takanawa, Tokyo, Japan.

\section{Received: 29 September 2014 Accepted: 8 February 2015}

\section{Published online: 21 February 2015}

\section{References}

1. Gallagher P, O'Mahony D. Inappropriate prescribing in older people. Rev Clin Gerontol. 2008;18(01):65.

2. Spinewine A, Schmader KE, Barber N, Hughes C, Lapane KL, Swine C, et al. Appropriate prescribing in elderly people: how well can it be measured and optimised? Lancet. 2007;370(9582):173-84.

3. Gallagher PF, Barry PJ, Ryan C, Hartigan I, O'Mahony D. Inappropriate prescribing in an acutely ill population of elderly patients as determined by Beers' Criteria. Age Ageing. 2008;37(1):96-101.

4. Chang C-M, Liu P-YY, Yang Y-HK, Yang Y-C, Wu C-F, Lu F-H. Use of the Beers criteria to predict adverse drug reactions among first-visit elderly outpatients. Pharmacotherapy. 2005;25(6):831-8.

5. Routledge PA, O'Mahony MS, Woodhouse KW. Adverse drug reactions in elderly patients. Br J Clin Pharmacol. 2004;57(2):121-6.

6. Lindley $\mathrm{CM}$, Tully MP, Paramsothy $\mathrm{V}$, Tallis RC. Inappropriate medication is a major cause of adverse drug reactions in elderly patients. Age Ageing. 1992;21(4):294-300.

7. Fick DM, Mion LC, Beers MH, Waller JL. Health outcomes associated with potentially inappropriate medication use in older adults. Res Nurs Health. 2008;31(1):42-51.

8. Passarelli MCG, Jacob-Filho W, Figueras A. Adverse drug reactions in an elderly hospitalised population: inappropriate prescription is a leading cause. Drugs Aging. 2005;22(9):767-77.

9. Schneeweiss S, Hasford J, Göttler M, Hoffmann A, Riethling A-K, Avorn J. Admissions caused by adverse drug events to internal medicine and emergency departments in hospitals: a longitudinal population-based study. Eur Jj Clin Pharmacol. 2002;58(4):285-91.

10. Klarin I, Wimo A, Fastbom J. The association of inappropriate drug use with hospitalisation and mortality: a population-based study of the very old. Drugs Aging. 2005;22(1):69-82.

11. Ebbesen J, Buajordet I, Erikssen J, Brørs O, Hilberg T, Svaar H, et al. Drugrelated deaths in a department of internal medicine. Arch Intern Med. 2001;161(19):2317-23.

12. Kripalani S, Henderson LE, Jacobson TA, Vaccarino V. Medication use among inner-city patients after hospital discharge: patient-reported barriers and solutions. Mayo Clin Proc. 2008;83(5):529-35.

13. Mansur N, Weiss A, Beloosesky Y. Is there an association between inappropriate prescription drug use and adherence in discharged elderly patients? Ann Pharmacother. 2009;43(2):177-84.

14. Fick DM, Waller JL, Maclean JR, Heuvel RV, Tadlock JG, Gottlieb M, et al. Potentially inappropriate medication use in a Medicare managed care population: Association with higher costs and utilization. J Manag Care Pharm. 2001;7(5):407-13.

15. Bates DW, Spell N, Cullen DJ, Burdick E, Laird N, Petersen LA, et al. The costs of adverse drug events in hospitalized patients adverse drug events prevention study group. JAMA. 1997;277(4):307-11.
16. Al-Omar HA, Al-Sultan MS, Abu-Auda HS. Prescribing of potentially inappropriate medications among the elderly population in an ambulatory care setting in a Saudi military hospital: trend and cost. Geriatr Gerontol Int. 2013;13(3):616-21.

17. Cahir C, Fahey T, Teeling M, Teljeur C, Feely J, Bennett K. Potentially inappropriate prescribing and cost outcomes for older people: a national population study. Br J Clin Pharmacol. 2010;69(5):543-52.

18. Bradley MC, Fahey T, Cahir C, Bennett K, O'Reilly D, Parsons C, et al. Potentially inappropriate prescribing and cost outcomes for older people: a cross-sectional study using the Northern Ireland Enhanced Prescribing Database. Eur J Clin Pharmacol. 2012;68(10):1425-33.

19. Hamano J, Tokuda Y. Inappropriate prescribing among elderly home care patients in Japan: prevalence and risk factors. J Prim Care Community Health. 2014;5(2):90-6.

20. Kaufmann CP, Tremp R, Hersberger KE, Lampert ML. Inappropriate prescribing: a systematic overview of published assessment tools. Eur J Cli Pharmacol. 2013;70(1):1-11.

21. American Geriatrics Society. Updated Beers criteria for potentially inappropriate medication use in older adults. J Am Geriatr Soc. 2012;60 (4):616-31.

22. Gallagher P, Ryan C, Byrne S, Kennedy J, O'Mahony D. STOPP (Screening Tool of Older Person's Prescriptions) and START (Screening Tool to Alert doctors to Right Treatment) Consensus validation. Int J Clin Pharmacol Ther. 2008;46(2):72-83.

23. Lam MPS, Cheung BMY. The use of STOPP/START criteria as a screening tool for assessing the appropriateness of medications in the elderly population. Expert Rev Clin Pharmacol. 2012;5(2):187-97.

24. Naugler CT, Brymer C, Stolee P, Arcese ZA. Development and validation of an improving prescribing in the elderly tool. Can J Clin Pharmacol. 2000;7(2):103-7.

25. Curtain CM, Bindoff IK, Westbury $J \mathrm{~L}$, Peterson GM. A comparison of prescribing criteria when applied to older community-based patients. Drugs Aging. 2013;30(11):935-43.

26. Hill-Taylor B, Sketris I, Hayden J, Byrne S, O'Sullivan D, Christie R. Application of the STOPP/START criteria: a systematic review of the prevalence of potentially inappropriate prescribing in older adults, and evidence of clinical, humanistic and economic impact. J Clin Pharm Ther. 2013;38(5):360-72.

27. Milos V, Rekman E, Bondesson $\AA$, Eriksson T, Jakobsson U, Westerlund T, et al. Improving the quality of pharmacotherapy in elderly primary care patients through medication reviews: a randomised controlled study. Drugs Aging. 2013;30(4):235-46.

28. Gillespie U, Alassaad A, Hammarlund-Udenaes M, Mörlin C, Henrohn D, Bertilsson $\mathrm{M}$, et al. Effects of pharmacists' interventions on appropriateness of prescribing and evaluation of the instruments' (MAI, STOPP and STARTs') ability to predict hospitalization-analyses from a randomized controlled trial. PLoS One. 2013;8(5):e62401.

29. Bergqvist M, Ulfvarson J, Karlsson EA. Nurse-led medication reviews and the quality of drug treatment of elderly hospitalized patients. Eur J Clin Pharmacol. 2009;65(11):1089-96.

30. Finkers F, Maring JG, Boersma F, Taxis K. A study of medication reviews to identify drug-related problems of polypharmacy patients in the Dutch nursing home setting. J Clin Pharm Ther. 2007;32(5):469-76.

31. Lenaghan E, Holland R, Brooks A. Home-based medication review in a high risk elderly population in primary care-the POLYMED randomised controlled trial. Age Ageing. 2007;36(3):292-7.

32. Garrido MM. Propensity scores: a practical method for assessing treatment effects in pain and symptom management research. J Pain Symptom Manage. 2014;48(4):711-8. 